\title{
Relationship Between Classification of Fabellae and the Severity of Keen Osteoarthritis: A Relevant Study in Chinese Population
}

\section{Lei Zhang}

Affiliated Traditional Chinese Medicine Hospital of Southwest Medical University

\section{Youliang Wen}

School of Rehabilitation Medicine GanNan Medical University

Chunying He

School of Clinical Medicine of Integrated Traditional Chinese and Western Medicine, Southwest Medical University

\section{Yan Zeng}

Department of Nephrology, Affiliated Traditional Chinese Medicine Hospital of Southwest Medical University

Jiangqin Luo ( $\sim$ ljq8702@163.com )

Southwest Medical University

\section{Chunyan He}

Southwest Medical University

\section{Junqiu Wang}

Southwest Medical University

\section{Qi Feng}

Southwest Medical University

\section{Research article}

Keywords: Fabella, Keen osteoarthritis, Morphology, Classification

Posted Date: August 17th, 2020

DOI: https://doi.org/10.21203/rs.3.rs-56744/v1

License: (c) (i) This work is licensed under a Creative Commons Attribution 4.0 International License.

Read Full License 


\section{Relationship Between Classification of Fabellae and the Severity of}

Keen Osteoarthritis: A Relevant Study in Chinese Population

\section{Lei Zhang, ${ }^{\mathrm{a}, \mathrm{b}, \mathrm{c}, \mathrm{d}, 1}$, Youliang Wen ${ }^{\mathrm{e}, 1}$, Chunying He $\mathrm{H}^{\mathrm{f}, 1}$, Yan Zengg, ${ }^{\mathrm{g}, 1}$ Jiangqin Luo ${ }^{\mathrm{h} *}$,} Chunyan He, Junqiu Wangf, Qi Feng ${ }^{i}$

a Department of Orthopedics, Affiliated Traditional Chinese Medicine Hospital of Southwest Medical University, Sichuan Luzhou, China, 646000

${ }^{b}$ Center for Orthopedic Diseases Research, Affiliated Traditional Chinese Medicine Hospital of Southwest Medical University, Sichuan Luzhou, China, 646000

${ }^{c}$ Expert Workstation in Luzhou, Sichuan Luzhou, China, 646000

${ }^{d}$ Clinical Base of Affiliated Traditional Chinese Medicine Hospital of Southwest Medical University, Guangdong Province Medical 3D Printing Application Transformation Engineering Technology Research Center, Sichuan Luzhou, China, 646000

e School of Rehabilitation Medicine GanNan Medical University, Jiangxi GanZhou China, 341000 .

${ }^{f}$ School of Clinical Medicine of Integrated Traditional Chinese and Western Medicine, Southwest Medical University, Sichuan Luzhou, China, 646000

${ }^{g}$ Department of Nephrology, Affiliated Traditional Chinese Medicine Hospital of Southwest Medical University, Luzhou, China, 646000

${ }^{h}$ Department of operating theatre, Affiliated Traditional Chinese Medicine Hospital of Southwest Medical University, Luzhou, China, 646000

${ }^{i}$ School of Clinical Medicine, Southwest Medical University, Sichuan Luzhou, China, 646000

${ }^{1}$ These authors contributed equally to this work.

* Corresponding author: Jiangqin Luo, Affiliated Traditional Chinese Medicine Hospital of Southwest Medical University, Luzhou, Sichuan province, China, 646000. E-mail addresses: 1jq8702@163.com.

\section{ABSTRACT}

Background: The fabella is a sesamoid bone having anatomical variations and it is 
more common in patients with primary keen osteoarthritis (KOA). The purpose of this study was to classify the fabellae and discuss the relationship between the classification of fabellae and the severity of KOA in Chinese.

Material and methods: 136 patients were measured and classified using CT three-dimensional reconstruction. According to the CT imaging characteristics, the fabellae were divided into 5 types: type I, a fabella on the lateral femoral condyle; type II, a fabella on the medial femoral condyle; type III, a fabella on the lateral femoral condyle and a fabella on the medial femoral condyle; type IV, two fabellae on the medial femoral condyle and type V, two fabellae on the lateral femoral condyle. The severity of KOA was assessed on the Recht grade by MRI. The data were analyzed with SPSS 24.0.

Results: The classification of fabellae were correlated with KOA grades $\left(\chi^{2}=35.026\right.$, $\mathrm{P}<0.05)$. In terms of KOA grades, grade I and grade II were occupied most of fabellar type II $(32,72.8 \%)$; type II and other types were significant statistical difference $(\mathrm{P}<0.05)$. Grade I and grade II were also the most of fabellar type IV (4, 100\%). Fabellar type V's biggest component were grade III and grade IV (6, 75\%). Type IV and type $\mathrm{V}$ were significant statistical difference $(\mathrm{P}<0.05)$.

Conclusion: The classification of fabellae were correlated with KOA grades. The type II may mean the lower KOA grades while type V may mean the higher KOA grades. Keywords: Fabella, Keen osteoarthritis, Morphology, Classification.

Trial registration: the Ethics Inspection Committee at Southwest Medical University, V1.0/20180801. Registered 20 August 2018.

\section{Introduction}

The fabella is a fibrocartilaginous or ossified sesamoid bone and because it often presented as a benign structure, the clinical significance of it usually was ignored ${ }^{[1]}$. However, under the mechanical stresses and loading, the fabella may act as a source of atypical knee pain in some cases, such as fabella syndrome, common fibular nerve palsy, chondromalacia, fabella dislocation, popliteal entrapment syndrome, and $\mathrm{KOA}^{[2-4]}$. The physicians may recognize it as an intra-articular loose body or an osteophyte, which could lead to delay in diagnosis and overuse of arthroscope $\mathrm{e}^{[5-7]}$. So, 
it is really important that we must put a premium on it. However, there were many studies focused on the fabellar prevalence but only a few studies reported anatomical morphology of fabella ${ }^{[8,9]}$.

The fabella has anatomical variations that could be located in the medial, lateral femoral condyle and embedded in the lateral head of gastrocnemius muscle mostly. Nevertheless, in recent years, some reports just described that fabella was located in the knee joint behind the lateral femoral condyle ${ }^{[10-13]}$. And the fabella has certainly anatomical variations in location and quantity but no one have classified ${ }^{[14,15]}$.

KOA is a degenerative and inflammatory joint disease which can lead to chronic pain and lower-limb disability ${ }^{[16]}$. KOA could causes serious socio-economic burdens, as reprot, the annual health care expenditures of KOA have been estimated at \$ US186 billion ${ }^{[17]}$. However, KOA affects articular cartilage mostly, the limited capacity of healing in articular cartilage indicates cannot be effectively repaired ${ }^{[18-20]}$. The relationship between KOA and fabellar occurrence rate has been supported. Several reviews have investigated that fabella was more common in patients with primary KOA. In their study, fabella was present in $35 \%$ of 300 patients with primary KOA and only in $15 \%$ of knees in the age-matched control group ${ }^{[21-23]}$. Pritchett JW et al speculated that in some way, fabella can predict KOA to provide more useful information for clinical use ${ }^{[24]}$. However, the link between the classification of fabellae and the severity of KOA remains unknown ${ }^{[25]}$.

In this study, the anatomical morphology of fabella and the types of fabella were performed in Chinese. And the relationship between classification of fabellae and the severity of KOA were analyzed.

\section{Material and methods}

\subsection{Ethical statement}

All the procedures were approved by the Ethics Committee of the Affiliated Traditional Chinese Medicine Hospital of Southwest Medical University (No. KY2018030) and all methods were performed in accordance with the relevant guidelines and regulations. All the measurements of fabella and KOA were collected at the Radiology Department of the Affiliated Traditional Chinese Medicine Hospital 
of Southwest Medical University (Luzhou, China).

\subsection{Instruments}

The KOA was measured by Magnetic resonance imaging acquisition (MAGNETON; Skyra, 3.0T) and its images were stored in the Picture Archiving Communication System (PACS; DJ Health Union Systems Corporation, Shanghai, China). After CT scanning (Somatom Emotion; Siemens AG, Munich, Germany), the images of fabella were reconstructed in 3D by syngoMMWP VE40B and all 3D images were stored in the Picture Archiving Communication System. PACS the software (UniReport version 2.0) can record, store a large of images and assist in accurate measuring.

\subsection{Subjects}

A total of 302 patients who had KOA was detected on 3.0T MRI at the Affiliated Traditional Chinese Medicine Hospital of Southwest Medical University. Informed consent was obtained from all subjects. But after the measuring by a spiral CT scanner, 136 patients who had fabella, KOA and met inclusion and exclusion criteria were included. They included 68 left sides and 68 right sides, 51 males and 85 females (mean age 62.71 \pm 10.75 years). Inclusion criteria:(1) According to the criteria of the American College of Rheumatology, primary KOA was diagnosed. (2) The fabella and KOA of each scan must be clear and intact. (3) The basic information and imaging data were complete. Exclusion criteria: (1) Previous knee injury or joint infection, such as patients with a history of systemic, rheumatic or inflammatory disease or chondrocalcinosis, hemochromatosis, inflammatory arthritis. (2) Patients who had contraindications for 3.0T MRI or CT.

\subsection{Method of measurement}

After acquiring the 3D reconstruction models of the fabella and MRI image of KOA, the measurement was made by 2 researchers (when there was a divergence, the third observer eventually decided) who engaged in the work of radiology more than three years. These researchers would take measurements all alone and each measurement was repeated three times, next averaging the three values obtained. The severity of KOA was assessed on the Recht grade ${ }^{[26]}$ (grade 0, normal cartilage; grade 
I, cartilage softening and/or swelling; grade II, mild surface fibrillation and/or less than $50 \%$ loss of cartilage thickness; grade III, severe surface fibrillation and/or loss of more than $50 \%$ of cartilage thickness but without exposure of subchondral bone; and grade IV, complete loss of cartilage with subchondral bone exposure). (Fig. 1)

According to $\mathrm{CT}$ imaging characteristics, the fabella was classified into five types based on the position, quantity. (Fig. 2).

(1) Type I: A fabella on the lateral femoral condyle.

(2) Type II: A fabella on the medial femoral condyle.

(3) Type III: A fabella on the lateral femoral condyle and a fabella on the medial femoral condyle.

(4) Type IV: Two fabellae on the medial femoral condyle.

(5) Type V: Two fabellae on the lateral femoral condyle.

The following parameters were defined and measured (accurate to $0.01 \mathrm{~cm}$ ) in the $3 \mathrm{D}$ reconstruction models.

Short axis: The short axis of fabella. (The fabellae with two were determined at a average value)

Long axis: The long axis of fabella. (The fabellae with two were determined at a average value)

A: The distance between two fabellae. (Fig. 3).

B: The distance between the proximal section of the femoral condyle and the section of the fabella. (Fig. 3).

\subsection{Statistical analysis}

Statistical analysis was performed by using SPSS, version 24.0 (IBM Corp, Armonk, NY, USA). All data were presented by the mean \pm standard deviation (SD). Categorical variables were recorded as numbers and percentages with frequency tables. The significance level was set at $\mathrm{P}=0.05$. One-way ANOVA, non-parametric tests and Shapiro-Wilk test were applied to analyze differences about the anatomic parameters of the fabella and classification. The differences in the fabellar classification and the severity of KOA was assessed using Conorer W. J test. The Spearman nonparametric correlation test was used for correlative analysis. 


\section{Results}

According to the location and quantity of fabellae, the fabellae were divided into 5 types: type I $(71,52.21 \%)$, type II $(44,32.35 \%)$, type III $(9,6.62 \%)$, type IV (4, $2.94 \%)$ and type $\mathrm{V}(8,5.88 \%)$. Among these classifications, type I was the most common while type IV was the lowest. The short axis of type III $(0.59 \pm 0.28 \mathrm{~cm})$ was significantly larger than type I $(0.45 \pm 0.19 \mathrm{~cm})$ and type II $(0.45 \pm 0.18 \mathrm{~cm})$, and the difference was statistically significant $(\mathrm{P}<0.05)$. With regard to long axis, type IV $(1.21 \pm 0.76 \mathrm{~cm})$ was significantly larger than other types, except for type III $(\mathrm{P}<0.05)$. Type III $(1.04 \pm 0.41 \mathrm{~cm})$ was larger than type I $(0.80 \pm 0.26 \mathrm{~cm})$ and type II $(0.80 \pm 0.35$ $\mathrm{cm})$, and there was significant difference $(\mathrm{P}<0.05)$. In term of $\mathrm{A}$ and $\mathrm{B}$, there were no significant statistical difference between different types $(\mathrm{P}>0.05)$. They were displayed on Table 1.

The classification of fabellae were correlated with KOA grades $\left(\chi^{2}=35.026\right.$, $\mathrm{P}<0.05)$. In terms of KOA grades, grade I and grade II were occupied most of type II (32, 72.8\%), type II and other types were significant statistical difference $(\mathrm{P}<0.05)$. Grade I and grade II were also the most of type IV (4, 100\%). Type V's biggest component were grade III and grade IV (6, 75\%). Type IV and type V were significant statistical difference $(\mathrm{P}<0.05)$. They were displayed on Table 2.

\section{Discussion}

Conventional radiography of the Kellgren-Lawrence stage division has been considered as a standard for describing the severity of $\mathrm{KOA}^{[27]}$. However, we choose the MRI of Recht grade as a result of it can assess soft tissue preferably and KOA affects the articular cartilage particularly ${ }^{[28,29]}$. The primary approaches currently available for KOA diagnosis are magnetic resonance imaging (MRI) which aids in diagnosing $\mathrm{KOA}$, determining $\mathrm{KOA}$ progression and prognosis, and monitoring treatment responses ${ }^{[30]}$. Using radiography alone to measure the loss of cartilage has limited clinical utility and only a modest correlation with symptom severity. Instead, MRI has consistently been manifested to have the capacity to be predictive of KOA symptoms ${ }^{[31]}$. Various studies demonstrated that MRI is highly specific and moderately sensitive and accurate for identifying articular cartilage degeneration of 
any severity, so it has become an essential research tool for KOA studies ${ }^{[32-34]}$.

The mean age of KOA is $62.71 \pm 10.75$ years and there are 51 males and 85 females which is consistent with what the published articles have reported that women have a higher prevalence of KOA and KOA primarily affects the elderly population worldwide ${ }^{[35,36]}$. Among these classifications, type I was the most common. The average range for short axis and long axis are $0.48 \pm 0.21 \mathrm{~cm}$ and $0.86 \pm 0.38 \mathrm{~cm}$, respectively. But some studies reported the fabella usually ranges from $0.5 \mathrm{~cm}$ to $2 \mathrm{~cm}$ in diameter which larger than Chinese. We hypothesized that this difference may based on races ${ }^{[37,38]}$. The short axis of type III $(0.59 \pm 0.28 \mathrm{~cm})$ was significantly larger than type I and type II $(\mathrm{P}<0.05)$. Concerning the long axis, type IV $(1.21 \pm 0.76 \mathrm{~cm})$ was significantly larger than other types, except for type III $(\mathrm{P}<0.05)$. Type III $(1.04 \pm 0.41 \mathrm{~cm})$ was larger than type I and type II $(\mathrm{P}<0.05)$. These results showed that the variability of the long and short axis between different types, we should pay attention to the difference when the fabella-related illness occurred. In terms of the distance between the proximal section of the femoral condyle and the section of the fabella, there was no significant statistical differences between different types $(\mathrm{P}>0.05)$. This demonstrated that the difference of $\mathrm{B}$ (The distance between the proximal section of the femoral condyle and the section of the fabella) is very little between different types and might be useful for localizing the fabella and scheduling the arthroscopic and surgical approach.

Regard to the treatment of fabella-related illness, it includes physical therapy, injection of local anesthetics or steroids around this bone, radial extracorporeal shock wave therapy or fabellectomy ${ }^{[39]}$. As fabella could cause KOA and it may be an atavistic pattern. Some people insisted, fabellae could be excised and found the posterolateral pain would disappear or greatly improve when removing the fabella ${ }^{[40]}$. Type V has corresponded to the higher grade of KOA. So, we speculated that if the imaging performance of fabella indicates type $\mathrm{V}$, we could predispose the fabella to prevent the occurrence and progression of KOA.

This study had some limitations. (1) As the prevalence of type III, IV, V was too low, this study's sample capacity was relatively limited. It would cause sampling bias. 
210 (2) Further studies on the mechanisms of the relationship between fabellar different

211 classification and the severity of KOA were encouraged.

\section{Conclusion}

213 According to the location and quantity of fabellae, the fabella was divided into 5 214 types and type I was the most common. The classification of fabellae were correlated 215 with KOA grades. The type II may mean the lower KOA grades while type V may 216 mean the higher KOA grades.

\section{Abbreviations}

218 KOA: keen osteoarthritis; A: The distance between two fabellae; B: The distance between the proximal section of the femoral condyle and the section of the fabella.

\section{Declarations}

\section{Ethics approval and consent to participate}

All the procedures were approved by the Ethics Inspection Committee at Southwest Medical University (No. KY2018030). All patient signed a General Consent of the Ethical Committee of Affiliated Traditional Chinese Medicine Hospital of Southwest Medical University for using and publishing their data for scientific use.

\section{Consent for publication}

All the authors agreed to publish.

\section{Availability of data and materials}

The datasets generated during and/or analysed during the current study are available from the corresponding author on reasonable request.

\section{Competing interests}

No conflict of interest exits in the submission of this manuscript, and the manuscript is approved by all authors for publication.

\section{Funding}

This work was supported by technological strategic cooperation project of southwest medical university, Luzhou Municipal people's Government (grant number. 2018LZXNYD-ZK43); the Affiliated Traditional Chinese Medicine Hospital of Southwest Medical University research project (grant number. 2019XYLH-001); the sub-items of Talent Team Project of Academician Zhong Shizhen, Southwest Medical University, Luzhou Municipal people's Government (grant number. 2018zszysrctdxm); 
the Expert Workstation Construction Project of Luzhou, Sichuan Province, China (grant number. 20180101) and the Scientific Innovation and Entrepreneurship Training Program of National Student (grant number. S201910793162).

\section{Authors' contributions}

LZ, YLW contribute to conception and design of study. Chunying $\mathrm{He}, \mathrm{YZ}$ contributions to write and editing this manuscript. JQL contribute to protocol and project development of study. Chunyan He, JQW and QF contribute to data collection and literature search. All authors read and approved the final manuscript.

\section{Acknowledgements}

The authors wanted to show their gratitude to the imaging department in Southwest Medical University that provided the samples.

\section{References}

[1] Hauser NH, Hoechel S, Toranelli M, et al. 2015. Functional and structural details about the fabella: what the important stabilizer looks like in the Central European population. Biomed Res Int. 343728.

[2] Usmani S, Marafi F, Ahmed N, et al. 2017. 18F-NaF PET-CT in Symptomatic Fabella Syndrome. Clin Nucl Med. 42(4), e199-e201.

[3] Ehara S. Potentially symptomatic fabella: MR imaging review. 2014. Jpn J Radiol. 32(1), 1-5.

[4] Cesmebasi A, Spinner RJ, Smith J, et al. 2016. Role of Sonography in the Diagnosis and Treatment of Common Peroneal Neuropathy Secondary to Fabellae. J Ultrasound Med. 35(2), 441-447.

[5] Provencher MT, Sanchez G, Ferrari MB, et al. 2017. Arthroscopy-Assisted Fabella Excision: Surgical Technique. Arthroscopy techniques. 6(2), e369-e374.

[6] Driessen A, Balke M, Offerhaus C, et al. 2014. The fabella syndrome - a rare cause of posterolateral knee pain: a review of the literature and two case reports. BMC Musculoskelet Disord. 15, 100.

[7] Franceschi F, Longo UG, Ruzzini L, et al. 2007. Dislocation of an enlarged fabella as uncommon cause of knee pain: a case report. Knee. 14(4), 330-332.

[8] Berthaume MA, Di Federico E, Bull AMJ. 2019. Fabella prevalence rate increases over 150 years, and rates of other sesamoid bones remain constant: a systematic review. J Anat, 2019, 17. 
[9] Pop TS, Pop AM, Olah P, et al. 2018. Prevalence of the fabella and its association with pain in the posterolateral corner of the knee: A cross-sectional study in a Romanian population. Medicine (Baltimore). 97(47), e13333.

[10] Tabira Y, Saga T, Takahashi N, et al. 2013. Influence of a fabella in the gastrocnemius muscle on the common fibular nerve in Japanese subjects. Clin Anat. 26(7), 893-902.

[11] $\mathrm{Wu} \mathrm{XD}, \mathrm{Yu}$ JH, Zou T, et al. 2017. Anatomical Characteristics and Biomechanical Properties of the Oblique Popliteal Ligament. Sci Rep. 7, 42698.

[12] Kaplan EB. 1961. The Fabellofibular and Short Lateral Ligaments of the Knee Joint. J Bone Joint Surg Am. 43-A, 169-179.

[13] Kawashima T, Takeishi H, Yoshitomi S, et al. 2007. Anatomical study of the fabella, fabellar complex and its clinical implications. Surg Radiol Anat. 29(8), 611-616.

[14] Zeng S-X, Dong X-L, Dang R-S, et al. 2012. Anatomic study of fabella and its surrounding structures in a Chinese population. Surg Radiol Anat, 34, 65-71.

[15] Decard BF, Nagy S, Garcia M, et al. 2017. An unusual case of bilateral peroneal palsies due to fabellae. Neurology. 88(9), 918.

[16] Wallace IJ, Worthington S, Felson DT, et al. 2017. Knee osteoarthritis has doubled in prevalence since the mid-20th century. Proc Natl Acad Sci U S A. 114(35): 9332-9336.

[17] Cross M, Smith E, Hoy D, et al. 2014. The global burden of hip and knee osteoarthritis: estimates from the global burden of disease 2010 study. Ann Rheum Dis. 73(7), 1323-1330.

[18] Altman RD. 2012. New guidelines for topical NSAIDs in the osteoarthritis treatment paradigm. Curr Med Res Opin. 26(12), 2871-2876.

[19] Shariatzadeh M, Song J, Wilson SL, et al. 2019. The efficacy of different sources of mesenchymal stem cells for the treatment of knee osteoarthritis. Cell Tissue Res. 378(3), 399-410.

[20] Meng Z, Huang R. 2018. Topical Treatment of Degenerative Knee Osteoarthritis. Am J Med Sci. 355(1), 6-12.

[21] Hou W, Xu L, Wang J, et al. 2019. Fabellar prevalence, degeneration and association with knee osteoarthritis in the Chinese population. Scientific reports. 9(1), 13046.

[22] Ando Y, Miyamoto Y, Tokimura F, et al. 2017. A case report on a very rare variant of popliteal artery entrapment syndrome due to an enlarged fabella associated with severe knee osteoarthritis. J Orthop Sci. 22(1), 164-168. 
[23] Prieto-Alhambra D, Judge A, Javaid MK, et al. 2014. Incidence and risk factors for clinically diagnosed knee, hip and hand osteoarthritis: influences of age, gender and osteoarthritis affecting other joints. Ann Rheum Dis. 73(9), 1659-64.

[24] Pritchett JW. 1984. The incidence of fabellae in osteoarthrosis of the knee. J Bone Joint Surg Am. 66(9), 1379-1380.

[25] Neogi T. 2013. The epidemiology and impact of pain in osteoarthritis. Osteoarthritis Cartilage. 21(9), 1145-53.

[26] Recht MP, Kramer J, Marcelis S, et al. 1993. Abnormalities of articular cartilage in the knee: analysis of available MR techniques. Radiology. 187(2), 473-478. [27] Jerban S, Chang EY, Du J. 2019. Magnetic resonance imaging (MRI) studies of knee joint osteoarthritis: a systematic review and meta-analysis. Osteoarthritis Cartilage. 20(1), 13-21. [29] Wright RW, MARS Group. 2014. Osteoarthritis Classification Scales: Interobserver under mechanical loading: Review. Magn Reson Imaging. 65, 27-36. [28] Menashe L, Hirko K, Losina E, et al. 2012. The diagnostic performance of MRI in Reliability and Arthroscopic Correlation. J Bone Joint Surg Am. 96(14), 1145-1151.

[30] Alizai H, Walter W, Khodarahmi I, et al. 2019. Cartilage Imaging in Osteoarthritis. Semin Musculoskelet Radiol. 23(5), 569-578.

[31] Kinds MB, Marijnissen AC, Bijlsma JW, et al. 2013. Quantitative radiographic features of early knee osteoarthritis: development over 5 years and relationship with symptoms in the CHECK cohort. J Rheumatol. 40(1), 58-65.

[32] Hayashi D, Roemer FW, Jarraya M. 2017. Imaging in Osteoarthritis. Radiologic clinics of North America. 55(5), 1085-1102.

[33] Demehri S, Guermazi A, Kwoh CK, et al. 2016. Diagnosis and Longitudinal Assessment of Osteoarthritis: Review of Available Imaging Techniques. Rheum Dis Clin North Am. 42(4), $607-620$.

[34] Miyauchi A, Kim-Kaneyama JR, Lei XF, et al. 2019. Alleviation of murine osteoarthritis by deletion of the focal adhesion mechanosensitive adapter, Hic-5. Sci Rep. 9(1), 15770.

[35] Quatman CE, Hettrich CM, Schmitt LC. 2011. The clinical utility and diagnostic performance of magnetic resonance imaging for identification of early and advanced knee osteoarthritis: a systematic review. The American journal of sports medicine. 39(7), 1557-1568. 
331 [36] Driban JB, Hootman JM, Sitler MR, et al. 2017. Is Participation in Certain Sports Associated 332 With Knee Osteoarthritis? A Systematic Review. J Ath1 Train. 52(6), 497-506.

333 [37] Rai MF, Duan X, Quirk JD, et al. 2017. Post-Traumatic Osteoarthritis in Mice Following 334 Mechanical Injury to the Synovial Joint. Sci Rep. 7, 45223.

335 [38] Eymard F, Chevalier X. 2019. Pharmacological treatments of knee osteoarthritis. Rev Prat. $336 \quad 69(5), 515-519$.

337 [39] Dalip D, Iwanaga J, Oskouian RJ, et al. 2018. A Comprehensive Review of the Fabella Bone. 338 Cureus. 10(6), e2736.

339 [40] Okano E, Yoshioka T, Yanai T, et al. 2016. Fabella syndrome as an uncommon cause of 340 posterolateral knee pain after total knee arthroplasty: a case report and review of the literature. 341 Case Rep Orthop. 2016, 4328462. 


\section{Figures}

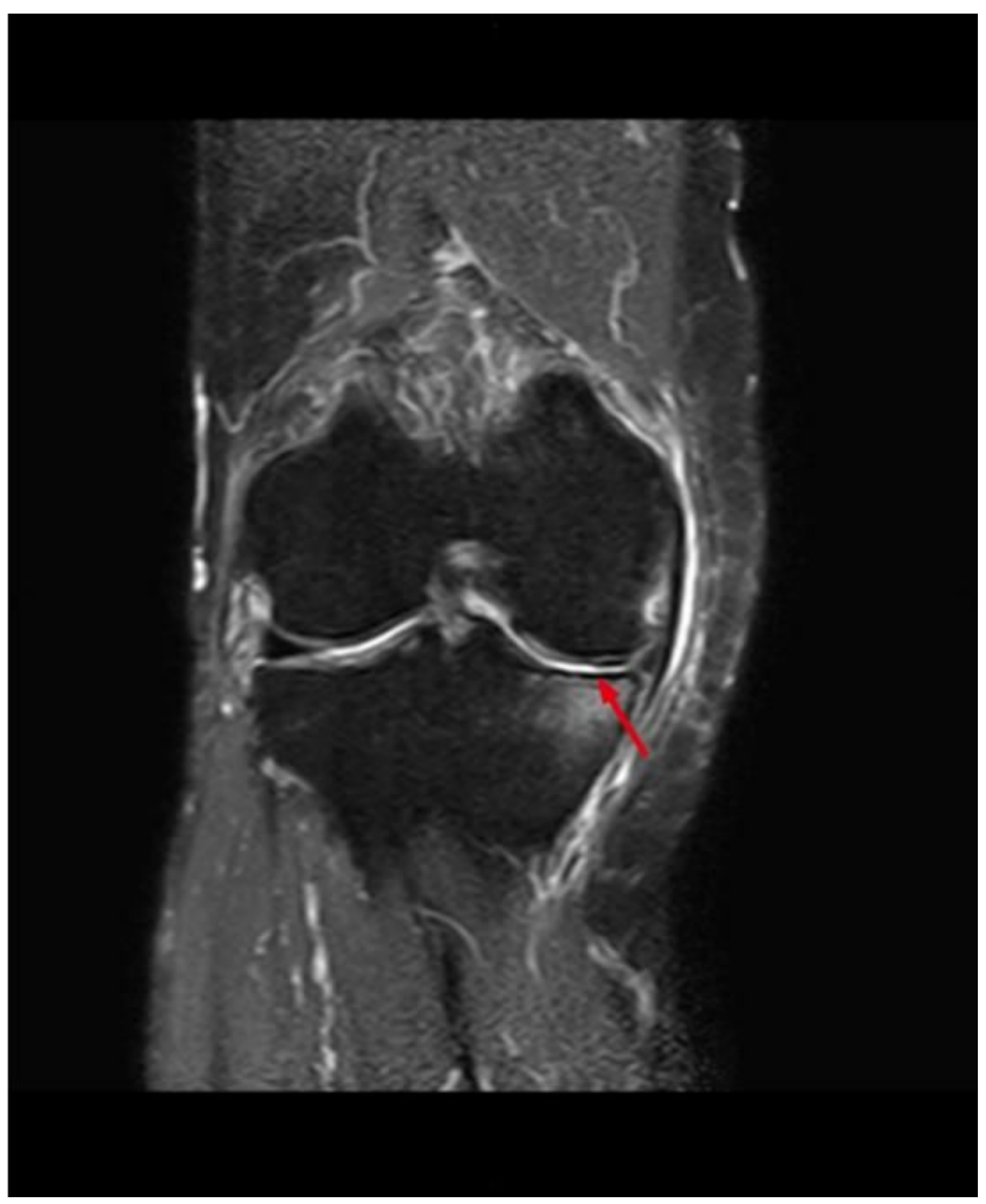

\section{Figure 1}

Measurement of the severity of KOA. Coronal intermediate-weighted fat suppressed MRI shows focal cartilage damage (red arrow). 


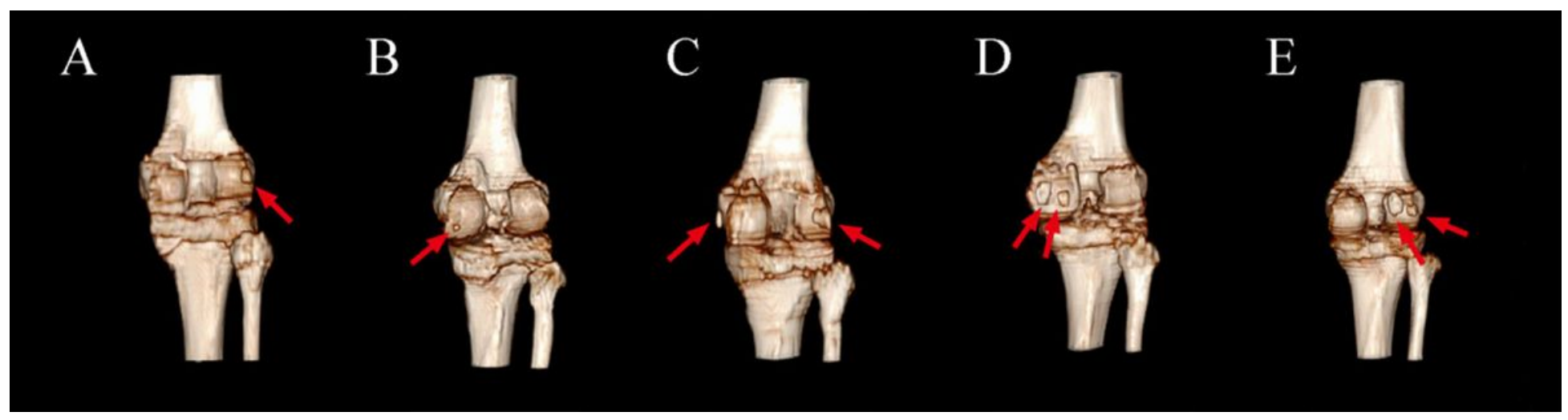

\section{Figure 2}

Various types of the fabellae. The fabella showed by red arrow. A: A fabella on the lateral femoral condyle. B: A fabella on the medial femoral condyle. C: A fabella on the lateral femoral condyle and a fabella on the medial femoral condyle. D: Two fabellae on the medial femoral condyle. E: Two fabellae on the lateral femoral condyle.

A

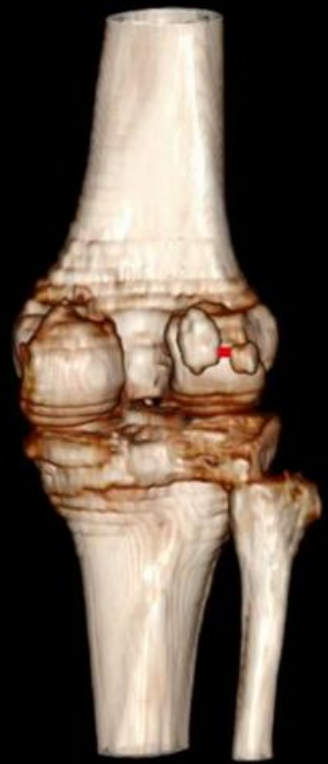

B

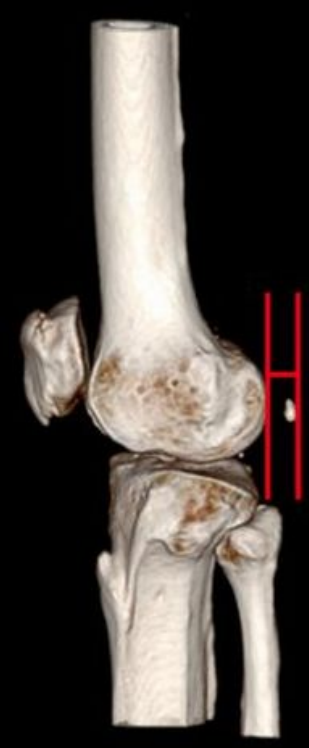

\section{Figure 3}

Measurement of the fabella. A The distance between two fabellae (red line). B The distance between the proximal section of the femoral condyle and the section of the fabella (red line). 